\title{
Traumatic Anterior Hip Dislocation in an Adolescent with an Associated Femoral Head "Hill-Sachs" Type Lesion
}

\section{Hiren M Divecha*, Ravi Badge, Niranjan Desai, Manzoor Sheikh and David Sochart}

Department of Orthopaedic Surgery, NorthManchester GeneralHospital, Delaunays Road, Crumpsall, Manchester, M8 5RB, UK

\begin{abstract}
Traumatic hip dislocations are uncommon injuries in the paediatric population, requiring urgent reduction to reduce the risk of avascular necrosis. Amongst other associated injuries, fractures of the femoral head, neck or acetabulum can occur. We present the case of a 14 year old boy who sustained a traumatic anterior obturator type hip dislocation with an associated supero-lateral "Hill-Sachs" type indentation osteochondral fracture of the femoral head. He was managed conservatively and by six weeks, was mobilising fully weight bearing unaided. At 17 month follow-up, he remained fully mobile with no complaints. Radiologically, the defect in the femoral head persisted with no evidence of collapse from avascular necrosis.

This type of osteochondral fracture associated with hip dislocation has been reported in the adult population, with varying reports of an increased risk of post-traumatic arthritis. The presented case highlights the important role of computed tomography (CT) in assessing these injuries. Furthermore, it brings to light a rare type of injury of the paediatric femoral head that warrants further long-term follow-up studies to determine the associated risk of avascular necrosis, hip instability and post-traumatic arthritis.
\end{abstract}

Keywords: Paediatric anterior hip dislocation; Indentation fracture; Osteochondral fracture; "Hill-Sachs" Lesion of femoral head

\section{Case Report}

A 14 year old boy sustained a left hip injury following a fall off a rope swing from a reported height of 6 meters. He was assessed using ATLS principles and his only injury involved the left hip. The clinical examination of the involved leg revealed a flexed, abducted and externally rotated lower limb. He was unable to move the hip joint and was tender on palpation with the femoral head palpable anteriorly in the groin region. He had no neurovascular injuries to the affected limb. Plain radiograph of his pelvis revealed an obturator type anterior hip joint dislocation (Figure 1). There were no obvious fractures of the femoral head or acetabulum noted.

Following urgent reduction under general anaesthetic, a satisfactory reduction was obtained with a stable, full range of motion. Following this, the patient was treated with bed rest, skin traction to the leg and adequate analgesia.

Post reduction AP pelvis and Judet radiographs confirmed a congruent reduction with no obvious fracture fragments. A Computed Tomography (CT) scan of the right hip with $3 \mathrm{D}$ reconstruction was performed to exclude the presence of any intra-articular fracture fragments. This confirmed a congruent reduction with no intraarticular bony fragments and no acetabular fracture. It did however reveal the presence of an indentation type fracture of the superolateral region of the femoral head and a small bony fragment lying at the obturator foramen (Figure 2). After further discussion with the musculoskeletal radiologist, it was felt that the small bony fragment lying at the obturator foramen was most likely a "push-off" fragment from the anterior acetabular rim.

The patient was managed conservatively and remained on bed rest with skin traction for one week after which he was allowed to mobilise partially weight bearing with the aid of crutches for a further five weeks. At six weeks post-injury, he was mobilising fully weight bearing with no discomfort and had a full, pain-free range of motion at the hip.
At 17 months post-injury,he remained fully mobile with no complaints of hip pain. Examination revealed a normal gait pattern and a full, pain-free range of motion at the hip. He had returned to sporting activities without any limitation. A follow-up CT scan with $3 \mathrm{D}$ reconstructions was performed (at 14 months post-injury) (Figure 3 ), which showed the persistence of the indentation fracture of the supero-lateral femoral head. This seems to be smaller in size and more shallow, possibly indicating some healing (diameter $=1.5 \mathrm{~cm}$ at followup vs $2 \mathrm{~cm}$ initial). Importantly, there was no collapse of the femoral head (a complication associated with hip dislocations due to avascular necrosis).

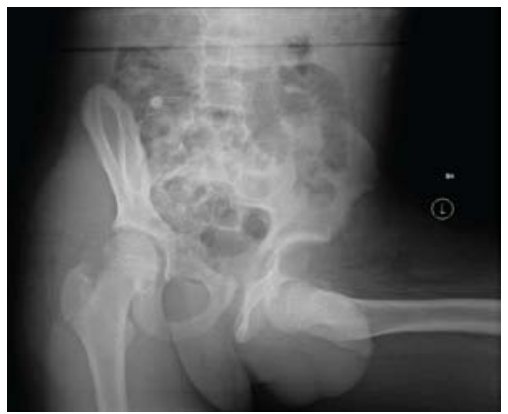

Figure 1: Pelvis $\mathrm{x}$-ray showing anterior obturator type hip joint dislocation.

*Corresponding author: Hiren M Divecha , Department of Orthopaedic Surgery, North Manchester General Hospital, Delaunays Road, Crumpsall, Manchester, M8 5RB, UK, E-mail: hiren.divecha@doctors.org.uk

Received January 30, 2011; Accepted February 13, 2012; Published February 20, 2012

Citation: Divecha HM, Badge R, Desai N, Sheikh M, Sochart D (2012) Traumatic Anterior Hip Dislocation in an Adolescent with an Associated Femoral Head "HillSachs" Type Lesion. J Trauma Treat 1:114. doi:10.4172/2167-1222.1000114

Copyright: (c) 2012 Divecha HM, et al. This is an open-access article distributed under the terms of the Creative Commons Attribution License, which permits unrestricted use, distribution, and reproduction in any medium, provided the original author and source are credited. 
Citation: Divecha HM, Badge R, Desai N, Sheikh M, Sochart D (2012) Traumatic Anterior Hip Dislocation in an Adolescent with an Associated Femoral Head "Hill-Sachs" Type Lesion. J Trauma Treat 1:114. doi:10.4172/2167-1222.1000114

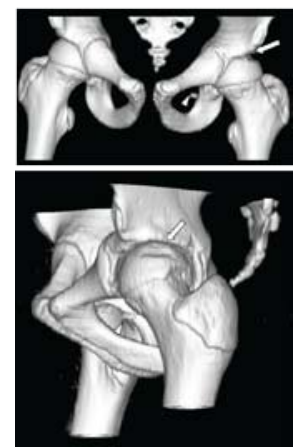

Figure 2: CT scan 3D reconstructions showing femoral head indentation lesion (white arrow) and bony fragment at obturator foramen (white arrow head).

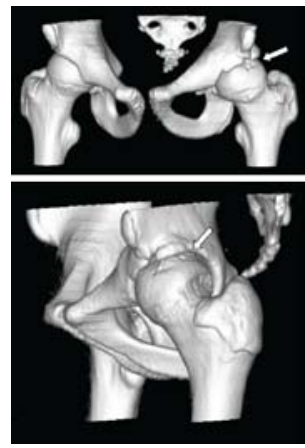

Figure 3: CT scan 3D reconstructions at 14 month follow-up showing persisting femoral head indentation lesion (white arrow).

\section{Discussion}

Hill and Sachs [1] described a compression fracture of the posterior humeral head caused by impaction of the glenoid rim following glenohumeral dislocation, the presence of which indicates shoulder instability. Birkett [2] was the first to describe a femoral head indentation injury associated with posterior dislocation in 1869. Funsten et al. [3] later described an indentation fracture of the femoral head associated with an anterior hip dislocation. Over the past few decades, several authors have described the presence of an indentation or "Hill-Sachs" type lesion of the femoral head found in $12-87 \%$ of traumatic hip dislocation in adults [4-8].

Paediatric traumatic hip dislocations are uncommon injuries. Approximately $5 \%$ of all traumatic hip dislocations occur in under- 14 year olds [9], with a male predominance (66-78\%) 10, 11. Posterior dislocations in children are 5-10 times more common than anterior dislocations [10-13].

Tehranzadeh et al. [8] analysed hip dislocations in 35 adult patients by CT. They found osteochondral impaction fractures in 63\% $(20$ of 32) of posterior dislocations and $100 \%$ of anterior dislocations (3 of 3). They suggested that these indentation fractures are usually unrecognisable on plain radiographs and sometimes overlooked on CT scan, especially with a chondral type of injury without significant subchondral involvement.

The only report of indentation fractures following traumatic hip dislocation in children was by Dussault et al. [5]. They reported eleven anterior dislocations in a mixed age group (10-56years). Eight of these had femoral head impaction fractures out of which five were osteochondral defects and three had localised femoral head flattening. Seven out of the eleven patients were followed-up and remained asymptomatic, with radiological evidence of healing in one indentation fracture. The authors did not specifically report on the short or longterm outcome of the paediatric case. Furthermore, CT scanning was not used to assess these injuries.

In summary, indentation fractures of the femoral head following traumatic hip dislocations in adults have been widely reported in the literature but not in paediatric cases. The identification of these injuries can be difficult on plain radiographs and may even be missed on CT scans [8]. The risk of post-traumatic arthritis associated with this injury seems to be high in adults (67\%) [4]. It remains uncertain whether the presence of such "Hill-Sachs" type indentation fractures of the femoral head in children is associated with a higher incidence of avascular necrosis, hip instability or post-traumatic arthritis.

We believe CT scanning with 3D reconstructions is an invaluable tool in the assessment of these injuries. At short-term follow-up (17 months) of this particular case, there did not seem to be any evidence of hip pain or instability and importantly, no evidence of avascular necrosis on repeat CT scan. The sequale of such injuries in the paediatric population remains unknown and a long-term follow-up of these injuries would be valuable in determining if there is a higher incidence of avascular necrosis, hip instability or post-traumatic arthritis.

\section{References}

1. Hill HA, Sachs MD (1940) The Grooved Defect of the Humeral Head: A Frequently Unrecognized Complication of Dislocations of the Shoulder Joint. Radiology 35: 690-700

2. Birkett $J$ (1869) Description of a dislocation of a head of a femur complicated with its fracture. Med Chir Trans 52:133-138

3. Funsten RV, Kinser P, Frankell CJ (1938) DASHBOARD DISLOCATION OF THE HIP A Report of Twenty Cases of Traumatic Dislocation. The Journal of Bone \& Joint Surgery 20: 124-132.

4. DeLee JC, Evans JA, Thomas J (1980) Anterior dislocation of the hip and associated femoral-head fractures. J Bone Joint Surg Am 62: 960-964.

5. Dussault RG, Beauregard G, Fauteaux P, Laurin C, Boisjoiy A (1980) Femoral head defect following anterior hip dislocation. Radiology 135: 627-629.

6. Erb RE, Steele JR, Nance EP Jr, Edwards JR (1995) Traumatic anterio dislocation of the hip: spectrum of plain film and CT findings. AJR Am J Roentgenol 165: 1215-1219.

7. Richardson P, Young JW, Porter D (1990) detection of cortical fracture of the femoral head associated with posterior hip dislocation. AJR Am J Roentgenol 155: 93-94.

8. Tehranzadeh J, Vanarthos W, Pais MJ (1990) Osteochondral impaction of the femoral head associated with hip dislocation: CT study in 35 patients. AJR Am J Roentgenol 155: 1049-1052.

9. Reiger H, Penning D, Klein W, Grunert J (1991) Traumatic dislocation of the hip in young children. Arch Orthop Trauma Surg 110: 114-117.

10. Funk FJ (1962) Traumatic dislocation of the hip in children. The Journal of Bone \& Joint Surgery 44: 1135-1145

11. Pearson DE, Mann RJ (1973) Traumatic hip dislocation in children. Clin Orthop Relat Res 92: 189-195.

12. Hamilton PR, Broughton NS (1998) Traumatic hip dislocation in childhood. $J$ Pediatr Orthop18: 691-694.

13. Hougaard K, Thomsen PB (1989) Traumatic hip dislocation in children.Followup of 13 cases. Orthopedics 12: 375-378. 